\title{
Three-dimensional modeling of the asymmetric blast wave from the 2006 outburst of RS Ophiuchi: Early X-ray emission $\star$
}

\author{
S. Orlando ${ }^{1,2}$, J. J. Drake ${ }^{3}$, and J. M. Laming ${ }^{4}$
}

\author{
1 INAF - Osservatorio Astronomico di Palermo "G.S. Vaiana", Piazza del Parlamento 1, 90134 Palermo, Italy \\ e-mail: orlando@astropa.inaf.it \\ 2 Consorzio COMETA, via Santa Sofia 64, 95123 Catania, Italy \\ 3 Harvard-Smithsonian Center for Astrophysics, 60 Garden Street, Cambridge, MA 02138, USA \\ 4 Space Science Division, Naval Research Laboratory, Code 7674L, Washington DC 20375, USA
}

Received 1 May 2008 / Accepted 21 November 2008

\section{ABSTRACT}

\begin{abstract}
Context. Chandra/HETG observations of the recurrent nova RS Ophiuchi at day 13.9 of its 2006 outburst reveal a spectrum covering a large range in plasma temperature and characterized by asymmetric and blue-shifted emission lines (Nelson et al. 2008; Drake et al. 2008).

Aims. We investigate the origin of asymmetries and broadening of the emission lines observed with Chandra/HETG. We explore possible diagnostics of the early blast wave and of the circumstellar medium (CSM) in which the explosion occurred.

Methods. We perform 3D hydrodynamic simulations of the blast wave from the 2006 outburst, propagating through the inhomogeneous CSM. The model takes into account the thermal conduction (including the effects of heat flux saturation) and the radiative cooling. From the simulations, we synthesize the X-ray emission and derive the spectra as they would be observed with $C h a n d r a / H E T G$. Results. The simulated nova remnant is highly aspherical and the blast wave is efficiently collimated by the inhomogeneous CSM. Our model reproduces the observed X-ray emission in a natural way if the CSM in which the outburst occurred is characterized by an equatorial density enhancement. Our "best-fit" model predicts that most of the early X-ray emission originates from a small region propagating in the direction perpendicular to the line-of-sight and localized just behind the interaction front between the blast wave and the equatorial density enhancement. The model predicts asymmetric and blue-shifted line profiles remarkably similar to those observed. These asymmetries are due to substantial X-ray absorption of red-shifted emission by ejecta material.

Conclusions. The comparison of high quality data of Chandra/HETG with detailed hydrodynamic modeling has allowed us to unveil, for the first time, the details of the structure emitting in the X-ray band in early phases of the outburst evolution, contributing to a better understanding of the physics of interactions between nova blasts and CSM in recurrent novae. This may have implications for whether or not RS Ophiuchi is a type Ia SN progenitor system.
\end{abstract}

Key words. shock waves - stars: binaries: symbiotic - stars: individual: RS Oph - stars: novae, cataclysmic variables X-rays: binaries

\section{Introduction}

RS Ophiuchi (RS Oph) is a symbiotic recurrent nova that went into its latest outburst on 2006 February 12.83 UT (Narumi et al. 2006); previous outbursts were recorded in 1898, 1933, 1958, 1967, and $1985^{1}$ (see Rosino 1987; Rosino \& Iijima 1987, and references therein). RS Oph is thought to be a binary system, comprising a red giant star that does not fill its Roche lobe, and a white dwarf of mass near the Chandrasekhar limit (Dobrzycka \& Kenyon 1994; Shore et al. 1996; Fekel et al. 2000); the system has a period of $455.72 \pm 0.83$ days (Fekel et al. 2000). Current theory tells us that, in this class of binaries, the outbursts occur on the white dwarf due to thermonuclear runaway of hydrogen-rich material transferred from the companion red giant onto the surface of the white dwarf (e.g. Kato 1991; Anupama \& Mikołajewska 1999; Starrfield et al. 2000).

The strong interest in studying RS Oph originates mainly from the belief that recurrent novae are progenitors of type Ia

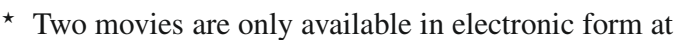
http://www . aanda.org

1 Two possible outbursts were also observed in 1907 (Schaefer 2004) and 1945 (Oppenheimer \& Mattei 1993).
}

supernovae ( $\mathrm{SNe} \mathrm{Ia}$ ). Two possible scenarios have been proposed: i) matter from the red giant is accreted by the white dwarf, causing the latter to increase in mass until it reaches the Chandrasekhar limit and explodes as an SN Ia; ii) two white dwarf stars merge, with the combined mass momentarily exceeding the Chandrasekhar limit, causing an SN Ia explosion. Which of these two scenarios is the more plausible is still under debate, so studying the nova outbursts from RS Oph can provide important insight into SNe Ia.

During the 2006 outburst an intensive international observing campaign was organized, incorporating observations ranging from radio to $\mathrm{X}$-ray wavelengths, and monitoring the outburst since the early phases of its evolution. The results included the detection of X-ray emission from hot gas, evolving from a dominant plasma temperature of $\approx 10 \mathrm{KeV}$ few days after the eruption (Sokoloski et al. 2006) to $\approx 4 \mathrm{KeV} 10$ days after optical maximum (Bode et al. 2006; Sokoloski et al. 2006). It has been suggested that bright X-ray emission arises from the shock-heated extended outer atmosphere of the red giant (e.g. Bode \& Kahn 1985; Bode et al. 2006; Nelson et al. 2008; Drake et al. 2008).

Chandra/HETG observations at day 13.9 revealed a rich spectrum of emission lines indicative of emitting plasma with 
temperatures ranging between 3 and $60 \mathrm{MK}$ (Nelson et al. 2008; Drake et al. 2008). Drake et al. (2008) noted that the lines are too strongly peaked to be explained by a sphericallysymmetric shock, suggesting a collimation mechanism of the $\mathrm{X}$-ray emitting plasma in the direction perpendicular to the lineof-sight. The lines also appear asymmetric and slightly blueshifted, while the red wings of the line profiles become weaker with increasing wavelength. Drake et al. (2008) suggested that the asymmetric nature of the circumstellar medium (CSM) in which the explosion occurred can be responsible for both the broad range in plasma temperature and the shock collimation observed.

Here we investigate the origin of the line asymmetries, broadening and blue-shifts observed with Chandra/HETG. We aim at exploring possible diagnostics of the early blast wave and of the inhomogeneous CSM in which the explosion occurred. To this end, we model the expansion of the blast wave from the 2006 outburst of the recurrent nova RS Oph through the extended outer atmosphere of the companion red giant, using detailed 3D hydrodynamic simulations. From the simulations we synthesize the X-ray emission and derive the spectra as they would be observed with Chandra/HETG.

In Sect. 2 we describe the hydrodynamic model, the numerical setup, and the synthesis of X-ray emission; in Sect. 3 we discuss the results; and finally in Sect. 4 we draw our conclusions.

\section{Model}

\subsection{Hydrodynamic modeling}

The blast wave is modeled by numerically solving the timedependent fluid equations of mass, momentum, and energy conservation in a $3 \mathrm{D}$ Cartesian coordinate system $(x, y, z)$, taking into account the radiative losses from an optically thin plasma and thermal conduction (including the effects of heat flux saturation):

$$
\begin{aligned}
& \frac{\partial \rho}{\partial t}+\nabla \cdot \rho \boldsymbol{u}=0 \\
& \frac{\partial \rho \boldsymbol{u}}{\partial t}+\nabla \cdot \rho \boldsymbol{u} \boldsymbol{u}+\nabla P=0 \\
& \frac{\partial \rho E}{\partial t}+\nabla \cdot(\rho E+P) \boldsymbol{u}=-\nabla \cdot q-n_{\mathrm{e}} n_{\mathrm{H}} \Lambda(T) \\
& \text { Here } \quad E=\epsilon+\frac{1}{2}|\boldsymbol{u}|^{2}
\end{aligned}
$$

is the total gas energy (internal energy, $\epsilon$, and kinetic energy), $t$ is the time, $\rho=\mu m_{H} n_{\mathrm{H}}$ is the mass density, $\mu=1.3$ is the mean atomic mass (assuming cosmic abundances), $m_{H}$ is the mass of the hydrogen atom, $n_{\mathrm{H}}$ is the hydrogen number density, $n_{\mathrm{e}}$ is the electron number density, $\boldsymbol{u}$ is the gas velocity, $T$ is the temperature, $q$ is the conductive flux, and $\Lambda(T)$ represents the radiative losses per unit emission measure (e.g. Raymond \& Smith 1977; Mewe et al. 1985; Kaastra \& Mewe 2000). We use the ideal gas law, $P=(\gamma-1) \rho \epsilon$, where $\gamma=5 / 3$ is the adiabatic index.

To allow for a smooth transition between the classical and saturated conduction regime, we followed Dalton \& Balbus (1993) and defined the conductive flux as (see also Orlando et al. 2005)

$$
q=\left(\frac{1}{q_{\text {spi }}}+\frac{1}{q_{\text {sat }}}\right)^{-1} .
$$

Here $q_{\text {spi }}$ represents the classical conductive flux (Spitzer 1962)

$$
q_{\mathrm{spi}}=-\delta_{\mathrm{T}} \epsilon 20\left(\frac{2}{\pi}\right)^{3 / 2} \frac{\left(k_{\mathrm{B}} T\right)^{5 / 2} k_{\mathrm{b}}}{m_{\mathrm{e}}^{1 / 2} \mathrm{e}^{4} Z \ln (\Lambda)} \nabla T
$$

where $k_{\mathrm{B}}$ is the Boltzmann constant, $m_{\mathrm{e}}$ is the electron mass, $e$ is the electron charge, $Z$ is the average atomic number, $\ln (\Lambda)$ is the Coulomb logarithm, $\delta_{\mathrm{T}}$ and $\epsilon$ parameters depend on the chemical composition, and in a proton-electron plasma: $\delta_{\mathrm{T}}=0.225$ and $\epsilon=0.419$. The saturated flux, $q_{\text {sat }}$, is (Cowie \& McKee 1977)

$q_{\mathrm{sat}}=-\operatorname{sign}(\nabla T) 5 \phi \rho c_{\mathrm{s}}^{3}$,

where $c_{\mathrm{S}}$ is the isothermal sound speed, and $\phi$ is a number on the order of unity. We set $\phi=0.3$ according to the values suggested for a fully ionized cosmic gas: $0.24<\phi<0.35$ (Giuliani 1984; Borkowski et al. 1989; Fadeyev et al. 2002, and references therein).

In order to trace the motion of the material ejected by the violent eruption, we consider a passive tracer associated with the ejecta. To this end, we add the equation

$\frac{\partial C_{\mathrm{cl}}}{\partial t}+\nabla \cdot C_{\mathrm{cl}} \boldsymbol{u}=0$

to the standard set of hydrodynamic equations. $C_{\mathrm{cl}}$ is the mass fraction of the ejecta inside the computational cell. The ejecta material is initialized with $C_{\mathrm{cl}}=1$, while $C_{\mathrm{cl}}=0$ in the ambient medium. During the shock evolution, the ejecta and the ambient medium mix together, leading to regions with $0<C_{\mathrm{cl}}<1$. At any time $t$ the density of ejecta material in a fluid cell is given by $\rho_{\mathrm{cl}}=\rho C_{\mathrm{cl}}$.

The calculations described in this paper were performed using FLASH, an adaptive mesh refinement multiphysics code (Fryxell et al. 2000). The hydrodynamic equations are solved using the FLASH implementation of the piecewice-parabolic method (Colella \& Woodward 1984). The code was designed to make efficient use of massively parallel computers using the message-passing interface (MPI) for interprocessor communications. The code has been extended with additional computational modules to handle radiative losses and thermal conduction (see Orlando et al. 2005, for the details of the implementation).

As an initial condition, we assume a spherical Sedov-type blast wave with radius $r_{\mathrm{b} 0}=1 / 3 \mathrm{AU}$ and with total energy $E_{\mathrm{b} 0}$, originating from the thermonuclear explosion on the white dwarf. The initial total energy of the explosion is partitioned so that $1 / 4$ of the energy is contained in thermal energy, and the other $3 / 4$ in kinetic energy. The initial total mass of the ejecta is $M_{\text {ej }}$. The blast propagates through the extended outer atmosphere (the wind) of the companion red giant and is off-set from the origin of the wind density distribution by $1.5 \mathrm{AU}$ (i.e. the system orbital separation; Dobrzycka \& Kenyon 1994). We follow O'Brien et al. (2006) and assume the gas density in the red giant wind proportional to $r^{-2}$ (where $r$ is the radial distance from the giant) and its temperature $2 \times 10^{4} \mathrm{~K}$. Note that the temperature values of the red giant wind (in symbiotic stars), $T_{\mathrm{w}}$, can range between few $10^{3} \mathrm{~K}$ up to $10^{5} \mathrm{~K}$ closer to the red giant (Friedjung et al. 1983). To evaluate the effects of $T_{\mathrm{w}}$ on the results of our simulations, we compared simulations with $T_{\mathrm{w}}=4000 \mathrm{~K}$ with simulations with $T_{\mathrm{w}}=20000 \mathrm{~K}$ and found that the results do not change.

In addition to the $r^{-2}$ density distribution, we include also an equatorial density enhancement (hereafter EDE) in the red giant wind, as suggested by VLBA radio synchrotron observations (O'Brien et al. 2006) and by HST observations 


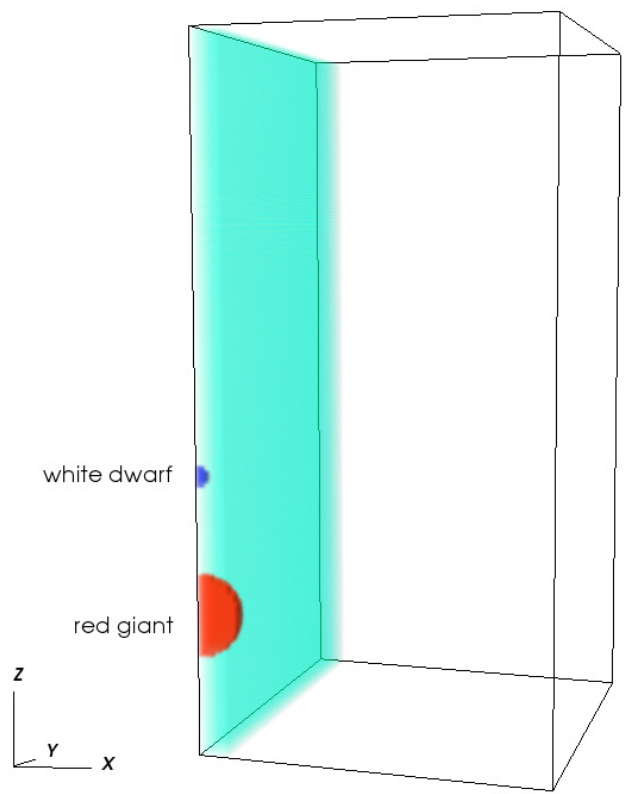

Fig. 1. Sketch of the initial geometry of the RS Oph system. The figure shows the quadrant of the spatial domain that is modeled numerically. The red giant is at the origin of the coordinate system, $(x, y, z)=$ $(0,0,0)$, and the computational domain extends $60 \mathrm{AU}$ in the $z$ direction, and $30 \mathrm{AU}$ in both the $x$ and $y$ directions; the white dwarf is located on the $z$ axis $(x=y=0)$ at $z=1.5 \mathrm{AU}$. The $(y, z)$ plane is the equatorial plane and the green structure marks the equatorial density enhancement.

(Bode et al. 2007) of the 2006 blast wave, and as predicted by detailed hydrodynamic modeling (Mastrodemos \& Morris 1999; Walder et al. 2008). The mass density distribution of the unperturbed CSM is given by:

$\rho=\frac{\rho_{\mathrm{w}}}{r_{\mathrm{au}}^{2}}+\rho_{\mathrm{eq}} \mathrm{e}^{-(h / L)^{2}}$,

where $\rho_{\mathrm{w}}=\mu m_{H} n_{\mathrm{w}}$ is the mass density at a distance of $1 \mathrm{AU}$ from the red giant, $r_{\mathrm{au}}$ is the radial distance from the giant in $\mathrm{AU}$, $\rho_{\mathrm{eq}}=\mu m_{H} n_{\mathrm{eq}}$ is the density enhancement at the equatorial plane, $h$ is the height above the equatorial plane and $L$ is a characteristic length scale.

In our $3 \mathrm{D}$ simulations, the symmetry of the problem allows us to solve the hydrodynamic equations in one quadrant of the spatial domain (see Fig. 1). The coordinate system is oriented in such a way that both the white dwarf and the red giant lie on the $z$ axis. The red giant is at the origin of the coordinate system, $(x, y, z)=(0,0,0)$, and the computational domain extends $60 \mathrm{AU}$ in the $z$ direction, and $30 \mathrm{AU}$ in both the $x$ and $y$ directions; the white dwarf is arbitrarily located to the north on the $z$ axis $(x=y=0)$ at $z=1.5$ AU (i.e. the system orbital separation; Dobrzycka \& Kenyon 1994).

At the coarsest resolution, the adaptive mesh algorithm used in the FLASH code (PARAMESH; MacNeice et al. 2000) uniformly covers the 3D computational domain with a mesh of $4 \times 4 \times 8$ blocks, each with $8^{3}$ cells. We allow for 8 nested levels of refinement during the first 3 days of evolution and for 6 levels in the rest of the simulation, with resolution increasing twice at each refinement level. The refinement criterion adopted (Löhner 1987) follows the changes in density, temperature, and tracer of ejecta. This grid configuration yields an effective resolution of $\approx 0.03 \mathrm{AU}$ at the finest level during the first 3 days
Table 1. Initial parameters of the 3D simulations.

\begin{tabular}{lccccc}
\hline \hline Run & $\begin{array}{c}M_{\mathrm{ej}}^{a} \\
{\left[M_{\odot}\right]}\end{array}$ & $\begin{array}{c}E_{\mathrm{b} 0}^{b} \\
{[\mathrm{erg}]}\end{array}$ & $\begin{array}{c}n_{\mathrm{w}}^{c} \\
{\left[\mathrm{~cm}^{-3}\right.}\end{array}$ & $\begin{array}{c}n_{\mathrm{eq}}^{d} \\
{\left[\mathrm{~cm}^{-3}\right.}\end{array}$ & $\begin{array}{c}L^{e} \\
{[\mathrm{AU}]}\end{array}$ \\
\hline ND-E43-N7 & $10^{-7}$ & $10^{43}$ & $2 \times 10^{7}$ & 0 & - \\
ND-E44-N7 & $10^{-6}$ & $10^{44}$ & $2 \times 10^{7}$ & 0 & - \\
ND-E43-N8 & $10^{-7}$ & $10^{43}$ & $2 \times 10^{8}$ & 0 & - \\
ND-E44-N8 & $10^{-6}$ & $10^{44}$ & $2 \times 10^{8}$ & 0 & - \\
ND-E43-N10 & $10^{-7}$ & $10^{43}$ & $2 \times 10^{10}$ & 0 & - \\
ND-E44-N10 & $10^{-6}$ & $10^{44}$ & $2 \times 10^{10}$ & 0 & - \\
YD-E43-N7-L2 & $10^{-7}$ & $10^{43}$ & $2 \times 10^{7}$ & $10^{8}$ & 2 \\
YD-E44-N7-L2 & $10^{-6}$ & $10^{44}$ & $2 \times 10^{7}$ & $10^{8}$ & 2 \\
YD-E43-N8-L2 & $10^{-7}$ & $10^{43}$ & $2 \times 10^{8}$ & $10^{8}$ & 2 \\
YD-E44-N8-L2 & $10^{-6}$ & $10^{44}$ & $2 \times 10^{8}$ & $10^{8}$ & 2 \\
YD-E44-N7-L1 & $10^{-6}$ & $10^{44}$ & $2 \times 10^{7}$ & $10^{8}$ & 1 \\
\hline
\end{tabular}

${ }^{a}$ Initial mass of ejecta; ${ }^{b}$ initial energy of the explosion; ${ }^{c}$ particle number density of the red giant wind at $1 \mathrm{AU} ;{ }^{d}$ particle number density enhancement at the equatorial plane; ${ }^{e}$ characteristic length scale in Eq. (6).

of evolution (when the radius of the nova remnant was $<5 \mathrm{AU}$ ) and $\approx 0.1 \mathrm{AU}$ at later times, corresponding to an equivalent uniform mesh of $1024 \times 1024 \times 2048$ and $256 \times 256 \times 512$ grid points, respectively. The higher spatial resolution during the first 3 days of evolution is required to describe properly the development of initially small structures dominated by radiative cooling. We use reflecting boundary conditions at $x=x_{\min }$ and $y=y_{\min }$ (consistent with the adopted symmetry) and zero-gradient conditions at the other boundaries.

We follow the expansion of the blast wave through the red giant wind for $\approx 15$ days, considering two sets of simulations: with or without the EDE. In both cases, we explore a parameter space defined by the initial mass of ejecta $M_{\mathrm{ej}}$ in the range $10^{-7}-10^{-6} M_{\odot}$, the initial energy of the explosion $E_{0}$ in the range $10^{43}-10^{44}$ erg (namely the published estimates for the ejected mass and energy of the explosion, e.g. Yaron et al. 2005; Sokoloski et al. 2006; O'Brien et al. 2006), and the particle number density of the red giant wind at 1 AU $n_{\mathrm{w}}$ in the range $2 \times 10^{7}-2 \times 10^{10} \mathrm{~cm}^{-3}$ (e.g. Dobrzycka \& Kenyon 1994). In the set of simulations with the EDE, we set $n_{\mathrm{eq}}=10^{8} \mathrm{~cm}^{-3}$ (consistent with the values found by Walder et al. 2008) and we explore the case of $L=1$ and 2 AU (see Eq. (6)). Table 1 summarizes the physical parameters characterizing the simulations.

\subsection{Synthesis of the $X$-ray emission}

From the model results, we synthesize the X-ray emission originating from the blast wave, applying a methodology analogous to the one described by Orlando et al. (2006) in the context of the study of supernova remnants (see also Miceli et al. 2006). The results of numerical simulations are the evolution of temperature, density, and velocity of the plasma in one quadrant of the whole spatial domain. We reconstruct the 3D spatial distribution of these physical quantities in the whole spatial domain, and we rotate the system about the $z$ axis in such a way that the inclination of the binary orbit to the line-of-sight $(\operatorname{LoS})$ is $35^{\circ}$, according to the values estimated from radial velocity observations before the 2006 outburst (Dobrzycka \& Kenyon 1994). Both the red giant and the white dwarf lie on the $z$ axis, and such a configuration assumes that the 2006 explosion occurred at quadrature, in agreement with the system ephemeris of Fekel et al. (2000) (see also O'Brien et al. 2006). 

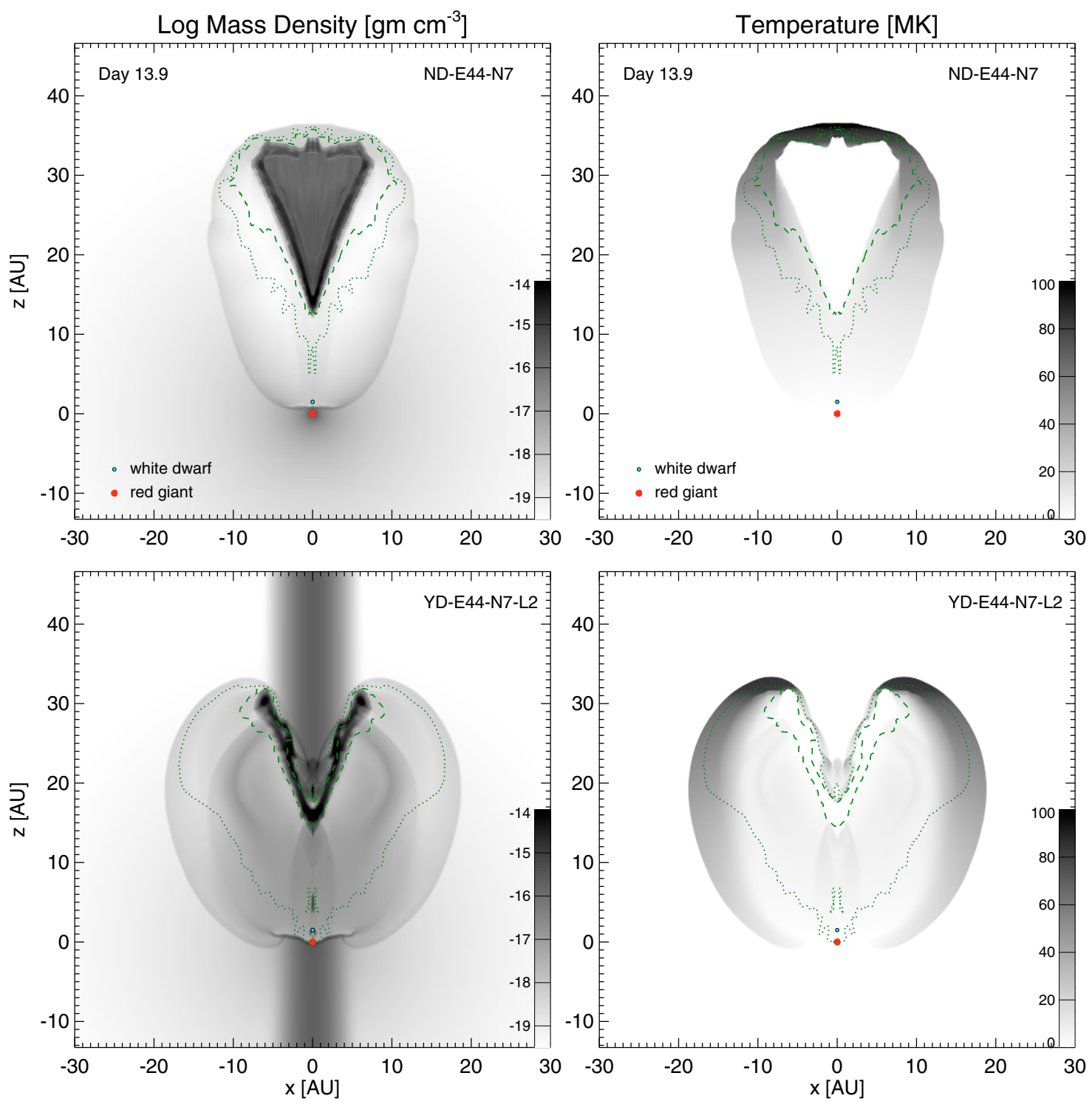

Fig. 2. 2D sections in the $(x, z)$ plane of the mass density distribution, in log scale, (on the left) and of the temperature (on the right) in the simulations ND-E44-N7 (upper panels) and YD-E44-N7-L2 (lower panels) at day 13.9. The EDE lies in the $(y, z)$ plane. The contours enclose zones whose content is made up of original ejecta material by more than $10 \%$ (dotted) and $90 \%$ (dashed).

The emission measure in the $j$ th domain cell is em $\mathrm{em}_{\mathrm{j}}=n_{\mathrm{Hj}}^{2} V_{\mathrm{j}}$ (where $n_{\mathrm{Hj}}^{2}$ is the hydrogen number density in the cell, $V_{\mathrm{j}}$ is the cell volume, and we assume fully ionized plasma). From the values of emission measure and temperature in the cell, we synthesize the corresponding X-ray spectrum, using the Astrophysical Plasma Emission Code (APEC; Smith et al. 2001) of hot collisionally ionized plasma. We assume solar metal abundances of Grevesse \& Sauval (1998, hereafter GS) for the CSM and abundances enhanced by $\times 10$ for the ejecta material (as suggested by Chandra/HETG observations; Drake et al. 2008). The spectral synthesis takes into account the thermal broadening of emission lines and the Doppler shift of lines due to the component of plasma velocity along the LoS.

The X-ray spectrum from each cell is filtered through the absorption column density relative to the position of the cell in the spatial domain. The absorption consists of two components: 1) the photoelectric absorption by the ISM, assuming a column density $N_{\mathrm{H}}=2.4 \times 10^{21} \mathrm{~cm}^{-2}$ (according to the value determined from $\mathrm{HI} 21 \mathrm{~cm}$ measurements, e.g. by
Hjellming et al. 1986, and consistent with a distance to RS Oph of $D_{\mathrm{oph}}=1.6 \mathrm{kpc}$, e.g. Bode 1987); and 2) the local absorption by the shocked CSM (with GS abundances) and by the ejecta (with GS abundances $\times 10$ ) encountered within the blast wave. The ISM, CSM, and ejecta absorption components are computed using the absorption cross-sections as a function of wavelength from Balucinska-Church \& McCammon (1992).

We integrate the absorbed X-ray spectra from the cells in the whole spatial domain. The resulting X-ray spectrum (originating from the whole blast wave) is then folded through the Chandra/HETG instrument response, considering the source at a distance $D_{\mathrm{oph}}=1.6 \mathrm{kpc}$. The exposure time is assumed to be $t_{\text {exp }}=10 \mathrm{ks}$ (the same value of the Chandra/HETG observations of RS Oph analyzed by Nelson et al. 2008; Drake et al. 2008).

\section{Results}

\subsection{Hydrodynamic evolution}

In all the models examined, we found the typical evolution of radiative shocks propagating through an inhomogeneous 

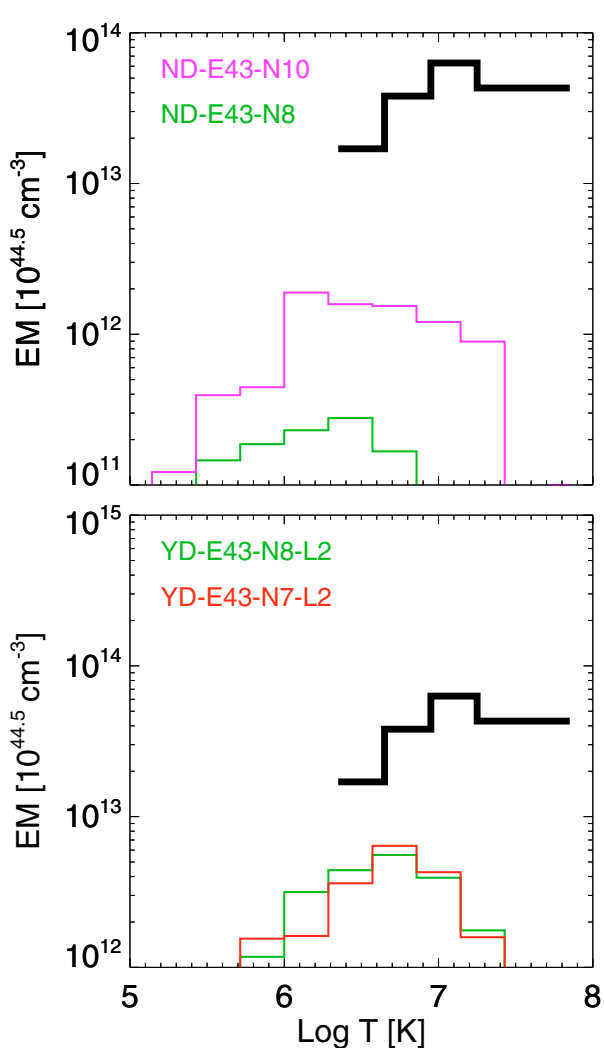

8
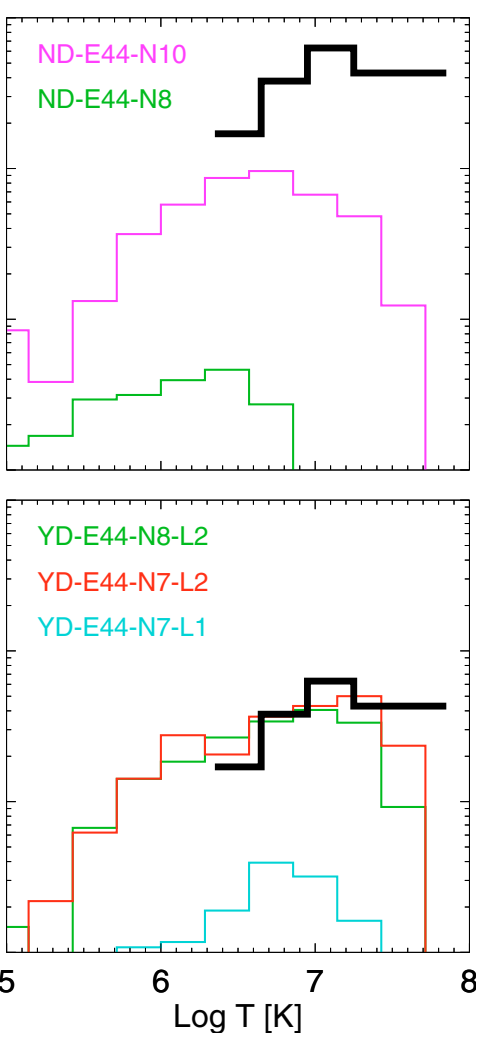

Fig. 3. Emission measure vs. temperature distribution, $\operatorname{EM}(T)$, of the blast wave at day 13.9. Upper panels show the result for models without EDE, lower panels for models with EDE. The black histogram represents the average $\operatorname{EM}(T)$ estimated from the analysis of Chandra/HETG observations (Drake et al. 2008). The factor $10^{44.5}$ assumes a distance for RS Oph of $1.6 \mathrm{kpc}$.

medium: the fast expansion of the shock front with temperatures of several millions degrees and the development of dense and cold regions dominated by radiative cooling, as the forward and reverse shocks progress through the CSM and ejecta, respectively. As examples, Fig. 2 shows $2 \mathrm{D}$ sections in the $(x, z)$ plane of the distributions of mass density (on the left) and of temperature (on the right) for the models ND-E44-N7 (i.e. without the EDE) and YD-E44-N7-L2 (with the EDE) at day 13.9.

The radiative cooling is very effective in the inner portion of the remnant (dark regions in left panels of Fig. 2) made up mostly of ejecta material. Hot post-shock regions are dominated by thermal conduction that contrasts the radiative cooling and suppresses hydrodynamic instabilities that would develop during the evolution of the blast wave (see Orlando et al. 2005). In particular, the thermal conduction suppresses the Rayleigh-Taylor instabilities that would develop at the contact discontinuity and that would drive the mixing of ejecta material with the shocked CSM. In our simulations, the mass mixing mainly comes from ejecta evaporation driven by thermal conduction rather than from hydrodynamic ablation (see Orlando et al. 2005, 2008). Figure 2 also shows the distribution of ejecta within the blast wave: regions whose content is made up of original ejecta material by more than $90 \%$ (dashed contours in the figure) are mostly dense, cold and dominated by radiative cooling, whereas regions made up of a mixture of ejecta and CSM are hot and dominated by thermal conduction (see right panels of Fig. 2). In all the cases, we found aspherical shock morphologies, with the blast wave propagating through inhomogeneous circumstellar gas distribution and being partially refracted around the red giant star.

In models without EDE, the aspherical morphology is due to the fact that the origin of the blast wave is off-set from the origin of the wind density distribution by $1.5 \mathrm{AU}$. This off-set is very effective in collimating the blast wave and ejecta. The shock front initially propagating away (toward) from the red giant goes through the $r^{-2}\left(r^{2}\right)$ density gradient and, as a consequence, its propagation velocity and temperature are high (small): the blast wave appears elongated in the direction away from the red giant and the hottest plasma component emitting in the X-ray band lies to the side away from the red giant (see upper panels of Fig. 2). The shock front propagating toward the red giant is strongly reflected by the high density medium and the resulting reflected shock contributes to sweep out the ejecta material away from the red giant (and from the white dwarf).

In models including the EDE, the aspherical shock morphology is quite complex and originates from the propagation of the shock through both the off-set red giant wind and the density enhancement at the equatorial plane (see also Girard \& Willson 1987; Walder et al. 2008). The latter component determines the collimation of the blast wave perpendicularly to the plane of the orbit of the central binary system and leads to a bipolar shock morphology distorted (by the off-set red giant wind) and converging on the side away from the red giant (see lower panels of Fig. 2). In this case, the hottest X-ray emitting plasma component lies in the two poles to the side away from the red giant (see lower right panel of Fig. 2). A movie showing the evolution of 3D spatial distribution of mass density, in log scale, for the model YD-E44-N7-L2 is provided as on-line material: the point of view is along the $y$ axis during the whole evolution, than it first rotates about the $x$ axis and finally about the $z$ axis up to an inclination of the binary orbit of $35^{\circ}$ (i.e. the value estimated before the 2006 outburst).

\subsection{Emission measure vs. temperature}

From the model results, we derived the $\operatorname{EM}(T)$ distribution of the blast wave. As discussed in Sect. 2.2, from the distribution 
of mass density, we derive the emission measure in the $j$ th domain cell as $\mathrm{em}_{\mathrm{j}}=n_{\mathrm{Hj}}^{2} V_{\mathrm{j}}$. The $\operatorname{EM}(T)$ distribution is then derived by binning the emission measure values into slots of temperature; the range of temperature $[4<\log T(\mathrm{~K})<8]$ is divided into 15 bins, all equal on a logarithmic scale. Figure 3 shows the $\operatorname{EM}(T)$ for models without EDE (upper panels), and with EDE (lower panels) at day 13.9, in the range of temperature $[5<\log T(\mathrm{~K})<8$ ], and assuming a distance for RS Oph of $1.6 \mathrm{kpc}$ (Bode et al. 2007). The figure also shows the average $\operatorname{EM}(T)$ estimated from resonance lines of abundant $\mathrm{He}$ - and H-like ions observed with Chandra/HETG (Drake et al. 2008).

In models without EDE, the shape of the $\operatorname{EM}(T)$ is characterized by a bump centered at temperatures between 1 and $5 \mathrm{MK}$ (see upper panels in Fig. 3). Increasing the initial energy of the explosion $E_{\mathrm{b} 0}$ from $10^{43}$ to $10^{44} \mathrm{erg}$ or increasing the density of the red giant wind at $1 \mathrm{AU}$ from $2 \times 10^{7}$ to $2 \times 10^{10} \mathrm{~cm}^{-3}$, increases the $\operatorname{EM}(T)$ distribution at all temperatures. However, in all the cases examined, the values of emission measure at temperatures around $10 \mathrm{MK}$ are orders of magnitude lower than those derived from the observations (Drake et al. 2008) and most of the shocked plasma is at temperatures below $5 \mathrm{MK}$. An initial energy of the explosion $E_{\mathrm{b} 0}>10^{45} \mathrm{erg}$ (and an ejected mass $M_{\mathrm{ej}}>10^{-5} M_{\odot}$ ) and/or a density of the red giant wind at $1 \mathrm{AU} n_{\mathrm{w}}>2 \times 10^{10} \mathrm{~cm}^{-3}$ (corresponding to a mass loss rate of $>5 \times 10^{-6} M_{\odot} \mathrm{yr}^{-1}$ for a wind velocity of $30 \mathrm{~km} \mathrm{~s}^{-1}$ ) would probably reproduce the observed emission measure, leading to a bump around $10 \mathrm{MK}$ in the $\operatorname{EM}(T)$ distribution. However, these values of $E_{\mathrm{b} 0}$ and $M_{\mathrm{ej}}$ are somewhat high for the true energy and ejected mass of the explosion (Yaron et al. 2005) and the value of $n_{\mathrm{w}}$ is also unrealistically large for the true density of the red giant wind. We conclude that models without the EDE fail to reproduce the values of emission measure derived from observations.

In models including the EDE, the emission measure at temperatures above $5 \mathrm{MK}$ is larger than that derived from the corresponding models without EDE. The shocked plasma of the EDE leads to a bump around $10 \mathrm{MK}$ in the $\operatorname{EM}(T)$ distribution (see lower panels in Fig. 3). The emission measure of the bump depends on the initial energy of the explosion $E_{\mathrm{b} 0}$. In models with $E_{\mathrm{b} 0}=10^{43} \mathrm{erg}$, the EM around $10 \mathrm{MK}$ is much lower than that derived from observations. Indeed the observed EM values can be reproduced with the models with $E_{\mathrm{b} 0}=10^{44} \mathrm{erg}$. The shape of the $\operatorname{EM}(T)$ distribution depends very slightly on the density of the red giant wind: decreasing $n_{\mathrm{w}}$, the EM slightly increases at temperatures above $\approx 10 \mathrm{MK}$. On the other hand, the $\operatorname{EM}(T)$ distribution strongly depends on the thickness of the EDE: the model with the thinnest EDE predicts EM values at $10 \mathrm{MK}$ well below those observed. We found that model YD-E44-N7-L2 is our "best-fit" model for reproducing the average $\operatorname{EM}(T)$ estimated from observations (see lower right panel in Fig. 3).

Note that our models underestimate the emission measure values observed at temperatures > 20 MK. The "observed" $\operatorname{EM}(T)$ distribution at high temperatures is mainly derived from the analysis of Fe XXV ( $\lambda 1.85)$, assuming GS abundances. However, Drake et al. (2008) noted that EM values derived from $\mathrm{Fe}$ ions appear to be anomalous and suggested that Fe probably suffers from effects of non-equilibrium ionization. In addition, as discussed in the next section, we found that a large contribution to the emission in the Fe XXV ( $\lambda 1.85)$ line comes from the shocked ejecta material, with GS abundances $\times 10$. As a consequence, the Fe XXV EM derived by Drake et al. (2008) would be overestimated.

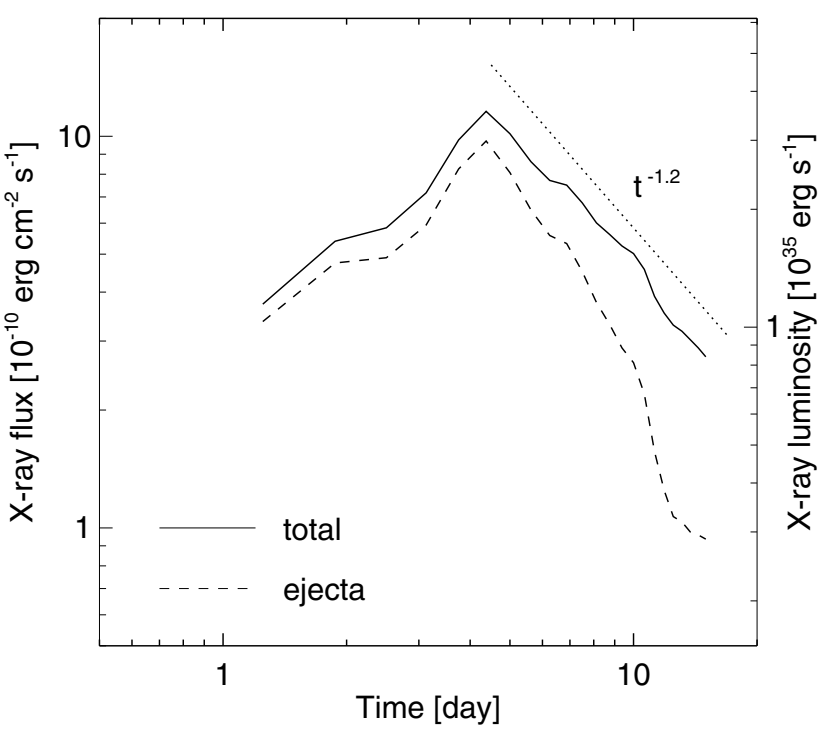

Fig. 4. X-ray light curves in the [0.6-12.4] keV band originating from the nova outburst (solid line) and from ejecta heated by the reverse shock (dashed line) for YD-E44-N7-L2. The X-ray flux is obtained by integrating the synthetic X-ray emission in the whole spatial domain and takes into account the effects of local absorption by shocked CSM and ejecta. The reference dotted line is a power law with index $\alpha=-1.2$. The $\mathrm{X}$-ray luminosity is reported on the right axis assuming a distance to RS Oph of $1.6 \mathrm{kpc}$.

\subsection{X-ray emission}

From the simulations, we synthesized the X-ray emission in the $[0.6$ - 12.4] $\mathrm{keV}$ band to match the observing range of Chandra/HETG, using the method outlined in Sect. 2.2. Figure 4 shows the X-ray light curve in this band for YD-E44-N7-L2 (i.e. our "best-fit" model), obtained by integrating the synthetic X-ray emission in the whole spatial domain and taking into account the effects of local absorption by shocked CSM and ejecta. The figure shows the X-ray flux, $F_{\mathrm{X}}$, originating from the nova outburst together with the contribution to X-rays from ejecta heated by the reverse shock (dashed line). The X-ray flux reaches its maximum quite early, around day 4 , and then decays as $t^{-1.2}$. The time of maximum X-ray emission is consistent with Swift/XRT observations (Bode et al. 2006), although the observed X-ray flux is slightly higher ${ }^{2}$ (by a factor $\approx 2$ ) than that derived from the simulation and decays as $t^{-1.5}$ (see also Sokoloski et al. 2006) instead of $t^{-1.2}$ as derived from the simulation. The X-ray decay rate is mainly driven by radiative cooling, which has a significant impact on the post-shock temperature structure (O'Brien \& Kahn 1987). The contribution to X-ray luminosity from shocked ejecta is rather important during the early phases of the evolution (more than $80 \%$ for $t<5$ days) and then decreases down to $34 \%$ at day 15 due to significant radiative cooling.

Figure 5 shows the map of X-ray emission integrated along the LoS and on pixels with size $\sim 0.1 \mathrm{AU}$ at day 13.9 for YD-E44-N7-L2. The map is drawn in linear (upper panel) and logarithmic (lower panel) scales to highlight structures with very different emission levels. The plane of the orbit of the central binary system lies on the $(y, z)$ plane and is assumed to be inclined by $35^{\circ}$ to the LoS, consistent with the inclination of the

\footnotetext{
2 However, note that the X-ray flux reported by Bode et al. (2006) is corrected for the effects of absorption by intervening material, whereas the synthesized X-ray flux takes into account the absorption by shocked CSM and ejecta.
} 

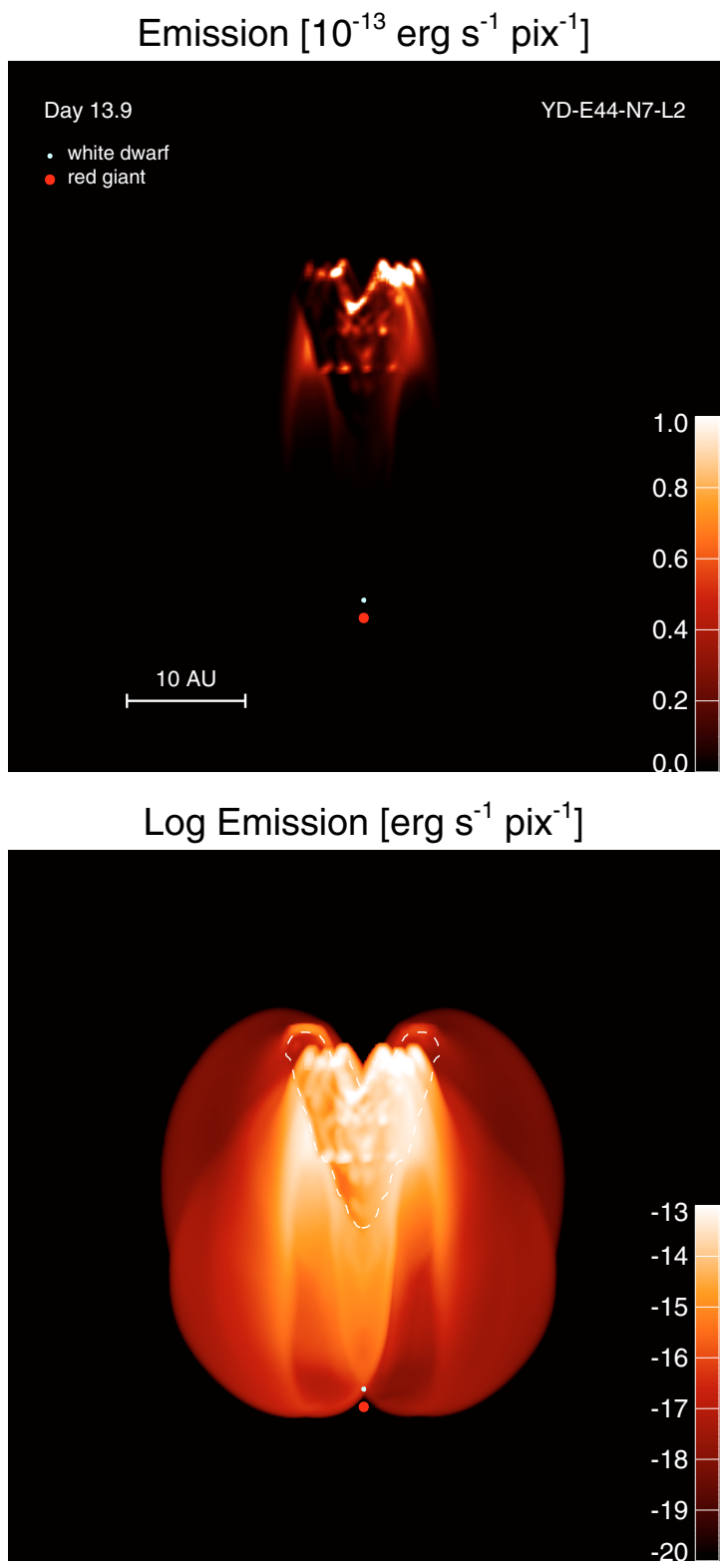

Fig. 5. X-ray images in the [0.6-12.4] keV band in linear (upper panel) and logarithmic (lower panel) scales derived from model YD-E44-N7L2 at day 13.9. The plane of the orbit of the central binary system lies on the $(y, z)$ plane and is assumed to be inclined by $35^{\circ}$ to the line-ofsight (Dobrzycka \& Kenyon 1994). The dashed contour encloses the ejecta material leading to the largest photoelectric absorption.

orbit from orbital solutions (Dobrzycka \& Kenyon 1994; see also Sect. 2.2). A movie showing the evolution of $X$-ray emission for YD-E44-N7-L2 is also provided as on-line material: the dotted gray line in the movie marks the position of the forward shock.

Figure 6 shows a 3D rendering of mass density, in log scale, at day 13.9 for YD-E44-N7-L2. We use two different color tables to identify the plasma with temperature larger (red color table) or lower (blue) than $1 \mathrm{MK}$. The volume has been clipped to show the internal structure of the blast wave. The comparison between Figs. 5 and 6 allows us to link X-ray emitting structures with plasma structures originating from the interaction of the blast wave with the inhomogeneous CSM.

Most of the X-ray emission originates from an irregular jetlike structure, lying on the $(y, z)$ plane, with a size of $\approx 10 \mathrm{AU}$ (see Fig. 5). The structure is located to the side away from the red giant, and the $\mathrm{X}$-ray emission is maximum in a region with size $\approx 4$ AU. By comparing the X-ray images in Fig. 5 with the $3 \mathrm{D}$ rendering of mass density in Fig. 6, we note that the X-ray emitting structure corresponds to a region just behind the interaction front between the blast wave and the EDE (yellow region in Fig. 6); the post-shock plasma at the equator is characterized by high density values $\left(10^{-15}<\rho<10^{-13} \mathrm{gm} \mathrm{cm}^{-3}\right)$ and temperatures ranging between 1 and $50 \mathrm{MK}$, with the highest values of density and temperature to the side away from the red giant.

The lower panel in Fig. 5 shows that faint X-ray emission also arises from a double-lobed structure with a size of $\approx 30 \mathrm{AU}$, surrounding the X-ray emitting jet. By comparing Figs. 5 and 6, we found that the double-lobed structure corresponds to regions downstream of the forward shock propagating perpendicularly to the plane of the binary orbit. These regions are characterized by density values ranging between $10^{-18} \mathrm{gm} \mathrm{cm}^{-3}$ and $10^{-17} \mathrm{gm} \mathrm{cm}^{-3}$ and temperatures between 10 and $85 \mathrm{MK}$. If the orientation of the EDE is the same as discussed by O'Brien et al. (2006), we speculate that this double-lobed structure roughly corresponds to the synchrotron-emitting partial shell observed in high-resolution radio observations (O'Brien et al. 2006; Rupen et al. 2008) although X-ray and radio brightness distributions may be not co-spatial ${ }^{3}$.

\subsubsection{Line profile analysis}

We integrated the emission of the whole spatial domain and derived the spectra for the HEG and MEG traces as predicted to be observed with the Chandra/HETG, in order to compare our analysis with the results obtained by Nelson et al. (2008) and by Drake et al. (2008). As expected, the synthetic spectra show emission lines from different elements, forming over a wide range of plasma temperatures, and reflecting the broad nature of the plasma temperature distribution discussed in Sect. 3.2.

Following Drake et al. (2008), we analyzed the line profiles of the most prominent spectral lines to investigate the origin of the broadening and asymmetries revealed in the Chandra/HETG observations (see, also, Nelson et al. 2008). Analogously to Drake et al. (2008), we restrict our analysis to HEG profiles, except in the case of O VIII ( $\lambda 18.97)$ which falls outside of the HEG range and is observed by the MEG. Table 2 summarizes the results of our analysis for the abundant $\mathrm{He}$ and $\mathrm{H}$-like ions, together with the Fe XVII ( $\lambda 15.01)$ resonance line. Figure 7 shows the line profiles for the abundant H-like ions Si XIV (ג6.18),

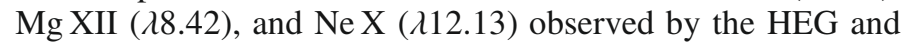
O VIII ( $\lambda 18.97)$ observed by the MEG. Note that the line profiles are affected by the instrument profile that is known to be more effective for decreasing wavelengths.

The line profiles exhibit broadening and asymmetries remarkably similar to those observed with Chandra/HETG. In velocity terms, most of the synthetic lines exhibit FWHM ranging between 1000 and $3000 \mathrm{~km} \mathrm{~s}^{-1}$ and FWZI ranging between 2500 and $8000 \mathrm{~km} \mathrm{~s}^{-1}$, in agreement with the results of Nelson et al. (2008) and Drake et al. (2008) (and similar to those observed in the IR by Das et al. 2006; Evans et al. 2007). The only exception is the bright He-like Fe XXV ( 11.85$)$ resonance line which exhibits much larger full-widths due to substantial contribution to the emission from the other two components of the Fe XXV

\footnotetext{
${ }^{3}$ Note that synchrotron radio brightness distributions also depend strongly on gradients of ambient magnetic field strength or of ambient plasma density (Orlando et al. 2007).
} 


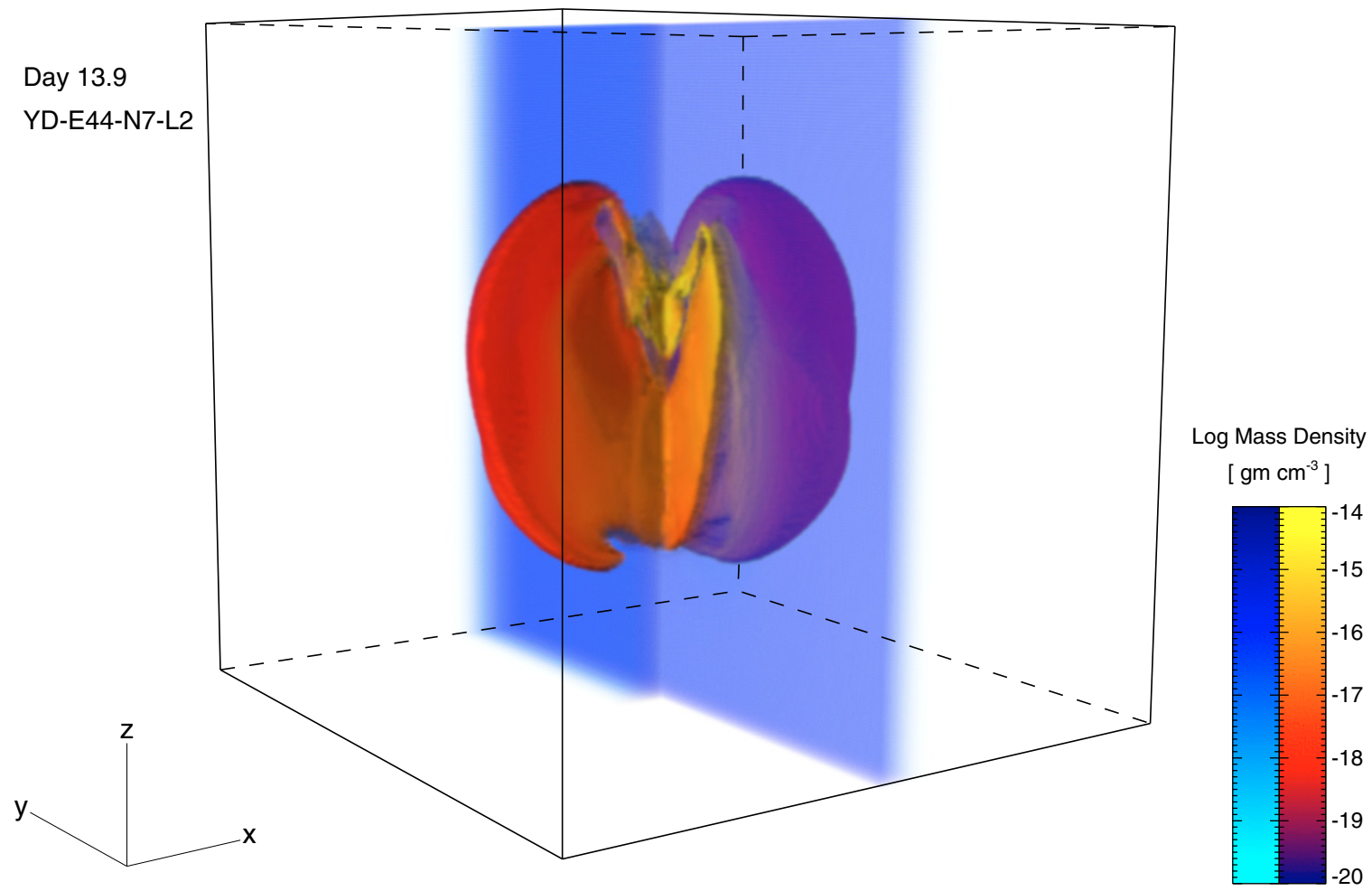

Fig. 6. Three-dimensional rendering of mass density, in log scale, at day 13.9 for the model YD-E44-N7-L2. One quarter of the volume has been removed to show the internal structure of the blast wave. Red (blue) color table marks the plasma with temperature larger (lower) than $1 \mathrm{MK}$. The plane of the orbit of the central binary system lies on the $(y, z)$ plane.

triplet at $\lambda \approx 1.86 \AA$ and $\lambda \approx 1.87 \AA$ and due to strong instrument effects.

Drake et al. (2008) noted that the observed line profiles are more peaked than expected for a spherically-symmetric shock and suggested that the emission is strongly enhanced in the direction of the plane of the sky. Our model predicts that most of the X-ray emission originates in a compact region propagating away from the red giant in the direction perpendicular to the LoS (see Fig. 5). As a result, we found that the line profiles are peaked as those observed.

In most of the lines, the centroids are systematically blueshifted and the amount of the shift depends on the wavelength

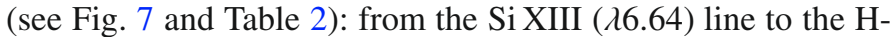
like O VIII ( 218.97$)$ doublet, the amount of blue-shift increases up to its maximum value. A similar trend has been found by Nelson et al. (2008) from the analysis of Chandra/HETG and XMM-Newton/RGS spectra of RS Oph at day 13.9, although the amount of blue-shift found by these authors is systematically higher than that predicted by our model. At variance with the other lines, the FeXXV ( $\lambda 1.85)$ resonance line exhibits a significant red-shift. Again, this different behavior is due to contribution to the emission from the other two components of the Fe XXV triplet.

As a result of the systematic blue-shift of emission lines, the line profiles tend to be more extended to the blue than the red (see Fig. 7 and the line profile blue-shift and red-shift at zero intensity in Table 2) with the only exception of the Fe XXV ( $\lambda 1.85)$ line. As in the Chandra/HETG spectra, BSZI and RSZI are observed to decrease with increasing wavelength, and this is particularly evident in the O VIII ( 218.97$)$ line profile in the MEG spectrum.
Table 2. Parameters (in units of $\left[\mathrm{km} \mathrm{s}^{-1}\right]$ ) characterizing the profiles of the most prominent spectral lines in the HEG and MEG spectra ${ }^{a}$.

\begin{tabular}{lrrrrr}
\hline \hline & $v_{\text {ctr }}^{b}$ & $F W H M^{c}$ & $F W Z I^{d}$ & \multicolumn{1}{c}{$B S Z I^{e}$} & $R_{S Z I^{f}}$ \\
\hline Fe XXV $(\lambda 1.85)$ & 1410 & 11351 & 29188 & 13183 & 16004 \\
S XVI $(\lambda 4.72)$ & -190 & 3172 & 8882 & 4631 & 4250 \\
S XV $(\lambda 5.03)$ & -30 & 2380 & 6843 & 3302 & 3540 \\
Si XIV $(\lambda 6.18)$ & -291 & 2183 & 5580 & 2959 & 2620 \\
Si XIII $(\lambda 6.64)$ & -203 & 1578 & 4510 & 2458 & 2052 \\
Mg XII $(\lambda 8.42)$ & -160 & 1246 & 3561 & 1941 & 1620 \\
Mg XI $(\lambda 9.16)$ & -196 & 981 & 2942 & 1667 & 1275 \\
NeX X $(\lambda 12.13)$ & -222 & 988 & 2471 & 1458 & 1013 \\
Fe XVII $(\lambda 15.01)$ & -309 & 499 & 1297 & 1008 & 289 \\
O VIII $(\lambda 18.97)$ & -355 & 553 & 1343 & 1066 & 276 \\
\hline
\end{tabular}

${ }^{a}$ Line profiles include the instrument profile that is known to be more effective for decreasing wavelengths; ${ }^{b}$ shift of the line centroid (negative values are for blue-shift); ${ }^{c}$ full widths at half maximum; ${ }^{d}$ full widths at zero intensity; ${ }^{e}$ line profile blue-shift at zero intensity; ${ }^{f}$ line profile red-shift at zero intensity.

The dashed contour in the lower panel in Fig. 5 encloses the ejecta material leading to the largest photoelectric absorption ${ }^{4}$. Given the geometry of the system and the inclination of the orbit, this material mostly absorbs the emission originating from the receding portion of the X-ray emitting jet-like structure (see also upper panel in Fig. 5) that contributes to the red-shifted emission. In fact, the figure shows an area of attenuated X-ray emission located in the left lobe of the bipolar structure. The resulting absorption of this component explains the asymmetry

\footnotetext{
${ }^{4}$ Equation (5) allows us to trace the ejecta material in the CSM.
} 


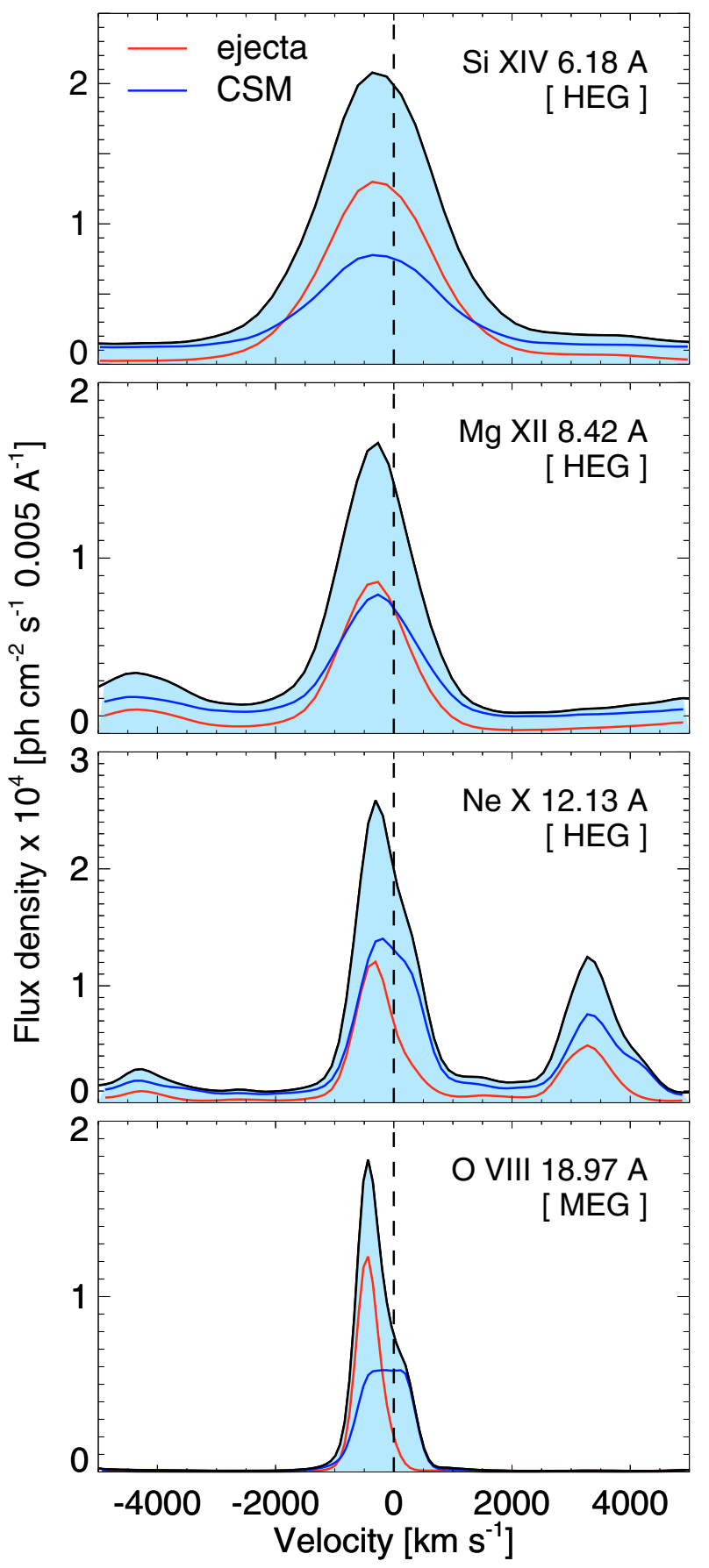

Fig. 7. Synthetic velocity profiles for the H-like resonance lines of $\mathrm{Si}$ XIV, Mg XII, Ne X, and O VIII derived from model YD-E44-N7-L2 at day 13.9. $\mathrm{Si}, \mathrm{Mg}$, and $\mathrm{Ne}$ lines are observed by the HEG, O line by the MEG. The figure also shows the contribution of shocked ejecta material (red lines) and shocked CSM (blue lines) to the X-ray emission.

and the systematic blue-shift of line profiles predicted by our model.

We also used the tracer associated with the ejecta to determine the contribution of shocked ejecta to the X-ray emission (see red lines in Fig. 7). We found that the red-shifted emission of the ejecta component is, in general, more absorbed than that of shocked CSM: the asymmetry of observed line profiles is mainly due to the ejecta component. We also found that, in general, the smaller the wavelength the larger is the contribution of shocked ejecta to the emission; in particular, the shocked ejecta contribute $\approx 60 \%$ to the emission of Fe XXV ( $\lambda 1.85)$ line and $\approx 40 \%$ to the emission of Fe XVII ( $\lambda 15.01)$ line. The different contributions of ejecta to X-ray emission (together with possible deviations from equilibrium of ionization) could explain the anomalous EM values derived from Fe ions by Drake et al. (2008). Among the lines analyzed, that with the largest contri-

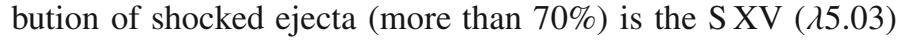
line.

\section{Summary and conclusions}

We have investigated through detailed hydrodynamic modeling the origin of asymmetries and broadening of the emission lines observed with Chandra/HETG during the 2006 outburst of RS Oph. To our knowledge, these simulations represent the first attempt to model the 3D structure of the blast wave originating from a nova outburst that simultaneously considers radiative cooling and thermal conduction (including heat flux saturation). Our findings have significant implications on the diagnostics of the blast wave during the early phases of evolution and lead to several useful conclusions:

1. In all the cases, we found that the nova remnant is highly aspherical, with the morphology of the blast wave deeply influenced by the pre-existing inhomogeneous CSM. Even in models without the EDE, the off-set of the nova explosion from the origin of the wind density distribution causes an effective collimation of the blast wave and ejecta.

2. Models without the EDE cannot reproduce the values of emission measure derived from Chandra/HETG observations unless the outburst energy is $E_{\mathrm{b} 0} \gg 10^{45} \mathrm{erg}$ (and the ejected mass in the explosion $M_{\mathrm{ej}} \gg 10^{-5} M_{\odot}$ ) and/or the density of the red giant wind is $n_{\mathrm{W}} \gg 10^{10} \mathrm{~cm}^{-3}$ at $1 \mathrm{AU}$; however, for the physical parameters characterizing RS Oph, these values seem too high for the true outburst energy and ejected mass in the explosion (Yaron et al. 2005) and for the density of the red giant wind (Dobrzycka \& Kenyon 1994). Models including the EDE reproduce in a natural way, without any further ad hoc assumption, the values of emission measure derived from Chandra/HETG observations if $E_{\mathrm{b} 0} \approx 10^{44}$ erg and $M_{\mathrm{ej}} \approx 10^{-6} M_{\odot}$.

3. Our "best-fit" model (YD-E44-N7-L2) predicts that, at day 13.9, most of the X-ray emission originates in a region with size $\approx 4 \mathrm{AU}$ localized at the interaction front between the blast wave and the EDE to the side away from the red giant; such an X-ray emitting region propagates in the direction perpendicular to the LoS. As a result, the synthetic line profiles are more peaked than expected for a sphericallysymmetric shock in nice agreement with the observations.

4. The synthetic line profiles are asymmetric and slightly blueshifted and they are remarkably similar to those observed. We found that the observed asymmetries are due to substantial X-ray absorption of red-shifted emission by ejecta material, confirming the conclusion of Drake et al. (2008).

5. Both shocked CSM and shocked ejecta contribute to the observed X-ray emission. The asymmetry and blue-shift of emission lines are mainly due to the shocked ejecta component which is more affected by X-ray absorption of redshifted emission. In general, the contribution of shocked ejecta to the X-ray emission decreases with increasing wavelength.

Our model shows that the broad range in the plasma temperature and the asymmetries of line profiles observed in Chandra/HETG 
spectra are due to the interaction of the blast wave with the preexisting inhomogeneous CSM. In addition, the asymmetric nature of the CSM into which the early blast wave is driven is also responsible of the apparent shock collimation in the plane of the sky. Note that the jet-like ejection emitting in the X-ray band predicted by our model does not coincide with the synchrotron jet observed in the radio band (O'Brien et al. 2006; Rupen et al. 2008): the former propagates in the plane of the orbit of the central binary system, whereas the latter propagates perpendicularly to the same plane.

Our analysis confirms that the comparison of high quality data with detailed hydrodynamic modeling can be a powerful tool to study and diagnose the physical properties of outbursts from recurrent novae and to interpret the observations. In particular, our results may provide useful constraints on the circumstellar gas distribution of RS Oph and suggest the existence of a dense region of the red giant wind at the equatorial plane. We also show that most of the early X-ray emission arises from this region as the blast wave from the 2006 outburst decelerates.

The scenario of an equatorial density enhancement is consistent with observations of the 2006 outburst in other wavelength bands, for instance VLBA radio synchrotron observations of an evolving ring-like structure (O'Brien et al. 2006; Rupen et al. 2008) and HST observations of a bipolar nebular structure (Bode et al. 2007). Also, the existence of the equatorial density enhancement may explain the presence of dust in the circumstellar environment in which the explosion occurs (Evans et al. 2007; Barry et al. 2008).

Our findings are also consistent with the asymmetric blast wave evolution described analytically by Girard \& Willson (1987) and with the results of hydrodynamic models describing the evolution of the circumstellar environment in binary systems (Mastrodemos \& Morris 1999; Walder et al. 2008). In particular, in systems comprising a red giant star, these models predict an equatorial density enhancement created by a spiral shock wave caused by the motion of the stars through the cool wind of the red giant; the enhancement is most pronounced in systems with the smallest binary separation, comparable to that in RS Oph.

It is worth noting that, in Walder et al. (2008), the blast wave appears more collimated perpendicularly to the plane of the orbit of the central binary system than in our simulations. The reason is that the CSM immediately around the white dwarf (within $1 / 3 \mathrm{AU}$ from the dwarf) is characterized by a rather complex density structure (see the lower right panel of Fig. 2 of Walder et al. 2008) determining the strong collimation of the blast wave, at the very beginning of the nova eruption (not described by our model). Since we assume the initial blast wave to be spherical (see Sect. 2.1), our model does not take into account such a strong initial collimation of the shock. To evaluate the effect of the initial condition on the evolution of the blast wave, we compared our "best-fit" model with an additional simulation with an identical setup but assuming an initial ellipsoid-shaped blast with the major axis perpendicular to the orbital plane. Such an initial condition describes the strong shock collimation occurred during the early phases of the nova eruption. Taking into account this initial shock collimation, we found that the shock expands very rapidly toward the perpendicular to the orbital plane and, after few days of evolution, its shape is similar to that found by Walder et al. (2008). Nevertheless, we also found that the main features characterizing the X-ray emission (broadening, asymmetries, blue-shift of emission lines) arising from the blast wave do not change qualitatively. Therefore, the main conclusions of this paper on the origin of the X-ray emission and of asymmetries and broadening of emission lines observed with Chandra/HETG remain.

The comparison of our model results with Chandra/HETG data suggests that the mass of ejecta in the 2006 outburst was of the order of $10^{-6} M_{\odot}$. From the analysis of the spectra collected with the Rossi X-ray Timing Explorer (RXTE), Sokoloski et al. (2006) derived the ejecta mass to be of a few times $10^{-7} M_{\odot}$ on the basis of some assumptions (time of transition to the SedovTaylor phase, shock speed during the ejecta-dominated phase, mass density inside the binary). However, taking into account the uncertainties in the determination of these values, the mass of ejecta derived by Sokoloski et al. (2006) can range between few times $10^{-7} M_{\odot}$ and few times $10^{-6} M_{\odot}$, i.e. a range of values consistent with that derived by our model.

Considering the mass of ejecta in the 2006 outburst of the order of $10^{-6} M_{\odot}$, it comes out that the white dwarf is increasing in mass if its growth rate is larger than $5 \times 10^{-8} M_{\odot} \mathrm{yr}^{-1}$. For lower values of the growth rate, the nova would throw off more mass than the white dwarf may have accreted in the intervening 22 years. Recently Hachisu et al. (2007) have estimated the white dwarf mass in RS Oph to be $1.35 \pm 0.01 M_{\odot}$ and its growth rate to be about $10^{-7} M_{\odot} \mathrm{yr}^{-1}$ in average (see also Kato et al. 2008). According to our results, therefore, we conclude that the white dwarf mass is effectively growing up and RS Oph could be the progenitor of a SN Ia as the white dwarf reaches the Chandrasekhar limit.

It will be interesting to expand the present study, including the ambient magnetic field, to investigate the evolution of the blast wave in later evolutionary phases and to make predictions on the synchrotron radio emission. The detailed comparison of model results with observations may lead to a major advance in the study of interactions between the blast wave and the magnetized CSM in recurrent novae, and may provide important insight into $\mathrm{SNe}$ Ia, of which recurrent novae are believed to be progenitors.

Acknowledgements. This work was supported in part by the Italian Ministry of University and Research (MIUR) and by Istituto Nazionale di Astrofisica (INAF). The software used in this work was in part developed by the DOEsupported ASC/Alliance Center for Astrophysical Thermonuclear Flashes at the University of Chicago, using modules for thermal conduction and optically thin radiation built at the Osservatorio Astronomico di Palermo. The simulations were executed on the Grid infrastructure of the Consorzio COMETA. This work makes use of results produced by the PI2S2 Project managed by the Consorzio COMETA, a project co-funded by the Italian Ministry of University and Research (MIUR) within the Piano Operativo Nazionale "Ricerca Scientifica, Sviluppo Tecnologico, Alta Formazione" (PON 20002006). More information is available at http://www.pi2s2.it and http:// www. consorzio-cometa.it.

\section{References}

Anupama, G. C., \& Mikołajewska, J. 1999, A\&A, 344, 177 Balucinska-Church, M., \& McCammon, D. 1992, ApJ, 400, 699

Barry, R. K., Danchi, W. C., Traub, W. A., et al. 2008, ApJ, 677, 1253

Bode, M. F. 1987, in RS Ophiuchi (1985) and the Recurrent Nova Phenomenon, ed. M. F. Bode, 241

Bode, M. F., \& Kahn, F. D. 1985, MNRAS, 217, 205

Bode, M. F., O’Brien, T. J., Osborne, J. P., et al. 2006, ApJ, 652, 629

Bode, M. F., Harman, D. J., O'Brien, T. J., et al. 2007, ApJ, 665, L63

Borkowski, K. J., Shull, J. M., \& McKee, C. F. 1989, ApJ, 336, 979

Colella, P., \& Woodward, P. 1984, JCP, 54, 174

Cowie, L. L., \& McKee, C. F. 1977, ApJ, 211, 135

Dalton, W. W., \& Balbus, S. A. 1993, ApJ, 404, 625

Das, R., Banerjee, D. P. K., \& Ashok, N. M. 2006, ApJ, 653, L141

Dobrzycka, D., \& Kenyon, S. J. 1994, AJ, 108, 2259

Drake, J., Laming, J., Ness, J.-U., et al. 2008, ApJ, in press

Evans, A., Kerr, T., Yang, B., et al. 2007, MNRAS, 374, L1 
Fadeyev, Y. A., Le Coroller, H., \& Gillet, D. 2002, A\&A, 392, 735

Fekel, F. C., Joyce, R. R., Hinkle, K. H., \& Skrutskie, M. F. 2000, AJ, 119, 1375 Friedjung, M., Stencel, R. E., \& Viotti, R. 1983, A\&A, 126, 407

Fryxell, B., Olson, K., Ricker, P., et al. 2000, ApJS, 131, 273

Girard, T., \& Willson, L. A. 1987, A\&A, 183, 247

Giuliani, J. L. 1984, ApJ, 277, 605

Grevesse, N., \& Sauval, A. J. 1998, Space Sci. Rev., 85, 161

Hachisu, I., Kato, M., \& Luna, G. J. M. 2007, ApJ, 659, L153

Hjellming, R. M., van Gorkom, J. H., Seaquist, E. R., et al. 1986, ApJ, 305, L71

Kaastra, J. S., \& Mewe, R. 2000, in Atomic Data Needs for X-ray Astronomy, 161

Kato, M. 1991, ApJ, 369, 471

Kato, M., Hachisu, I., \& Luna, G. J. M. 2008, ArXiv e-prints, 807

Löhner, R. 1987, Comp. Meth. Appl. Mech. Eng., 61, 323

MacNeice, P., Olson, K. M., Mobarry, C., de Fainchtein, R., \& Packer, C. 2000, Comp. Phys. Comm., 126, 330

Mastrodemos, N., \& Morris, M. 1999, ApJ, 523, 357

Mewe, R., Gronenschild, E. H. B. M., \& van den Oord, G. H. J. 1985, A\&AS, 62, 197

Miceli, M., Reale, F., Orlando, S., \& Bocchino, F. 2006, A\&A, 458, 213

Narumi, H., Hirosawa, K., Kanai, K., et al. 2006, IAU Circ., 8671, 2

Nelson, T., Orio, M., Cassinelli, J. P., et al. 2008, ApJ, 673, 1067

O'Brien, T. J., \& Kahn, F. D. 1987, MNRAS, 228, 277

O'Brien, T. J., Bode, M. F., Porcas, R. W., et al. 2006, Nature, 442, 279
Oppenheimer, B. D., \& Mattei, J. A. 1993, J. Amer. Association of Variable Star Observers (JAAVSO), 22, 105

Orlando, S., Peres, G., Reale, F., et al. 2005, A\&A, 444, 505

Orlando, S. Bocchino, F., Peres, G., Reale, F., Plewa, T., \& Rosner, R. 2006, A\&A, 457, 545

Orlando, S., Bocchino, F., Reale, F., Peres, G., \& Pagano, P. 2008, ApJ, 678, 274

Orlando, S., Bocchino, F., Reale, F., Peres, G., \& Petruk, O. 2007, A\&A, 470, 927

Raymond, J. C., \& Smith, B. W. 1977, ApJS, 35, 419

Rosino, L. 1987, in RS Ophiuchi (1985) and the Recurrent Nova Phenomenon, ed. M. F. Bode, 1

Rosino, L., \& Iijima, T. 1987, in RS Ophiuchi (1985) and the Recurrent Nova Phenomenon, ed. M. F. Bode, 27

Rupen, M. P., Mioduszewski, A. J., \& Sokoloski, J. L. 2008, ApJ, 688, 559

Schaefer, B. E. 2004, IAU Circ., 8396, 2

Shore, S. N., Kenyon, S. J., Starrfield, S., \& Sonneborn, G. 1996, ApJ, 456, 717 Smith, R. K., Brickhouse, N. S., Liedahl, D. A., \& Raymond, J. C. 2001, ApJ, 556, L91

Sokoloski, J. L., Luna, G. J. M., Mukai, K., \& Kenyon, S. J. 2006, Nature, 442, 276

Spitzer, L. 1962, Physics of Fully Ionized Gases (New York: Interscience, 1962) Starrfield, S., Truran, J. W., \& Sparks, W. M. 2000, New Astron. Rev., 44, 81

Walder, R., Folini, D., \& Shore, S. N. 2008, A\&A, 484, L9

Yaron, O., Prialnik, D., Shara, M. M., \& Kovetz, A. 2005, ApJ, 623, 398 